\title{
Resting State BOLD Variability of the Posterior Medial Temporal Lobe Correlates with Cognitive Performance in Older Adults with and without Risk for Cognitive Decline
}

\author{
Tyler J. Good, ${ }^{1,2}$ Joshua Villafuerte, ${ }^{1,2}$ - Jennifer D. Ryan, ${ }^{1,2,3}$ Cheryl L. Grady, ${ }^{1,2,3}$ and \\ Morgan D. Barense ${ }^{1,2}$
}

https://doi.org/10.1523/ENEURO.0290-19.2020

${ }^{1}$ Rotman Research Institute, Baycrest Health Sciences, Toronto M6A 2E1, Ontario, ${ }^{2}$ Department of Psychology, University of Toronto, Toronto M5S 3G3, Ontario, and ${ }^{3}$ Department of Psychiatry, University of Toronto, Toronto M5T 1R8, Ontario

\begin{abstract}
Local brain signal variability [SD of the BOLD signal $\left(\mathrm{SD}_{\mathrm{BOLD}}\right]$ ] correlates with age and cognitive performance, and recently differentiated Alzheimer's disease (AD) patients from healthy controls. However, it is unknown whether changes to $\mathrm{SD}_{\mathrm{BO}}$ precede diagnosis of $\mathrm{AD}$ or mild cognitive impairment. We compared ostensibly healthy older adult humans who scored below the recommended threshold on the Montreal cognitive assessment (MoCA) and who showed reduced medial temporal lobe (MTL) volume in a previous study ("at-risk" group, $n=20$ ), with healthy older adults who scored within the normal range on the MoCA ("control" group, $n=20$ ). Using multivariate partial least-squares analysis we assessed the correlations between $\mathrm{SD}_{\mathrm{BO}}$ and age, MoCA score, global fractional anisotropy, global mean diffusivity, and four cognitive factors. Greater $\mathrm{SD}_{\mathrm{BO}} \mathrm{LD}$ in the MTL and occipital cortex positively correlated with performance on cognitive control/speed tasks but negatively correlated with memory scores in the control group. These relations were weaker in the at-risk group. A post hoc analysis assessed associations between MTL volumes and $\mathrm{SD}_{\mathrm{BOLD}}$ in both groups. This revealed a negative correlation, most robust in the at-risk group, between MTL $S D_{\mathrm{BO}}$ and MTL subregion volumetry, particularly the entorhinal and parahippocampal regions. Together, these results suggest that the association between $\mathrm{SD}_{\mathrm{BOLD}}$ and cognition differs between the at-risk and control groups, which may be because of lower MTL volumes in the at-risk group. Our data indicate relations between MTL $\mathrm{SD}_{\mathrm{BO}}$ and cognition may be helpful in understanding brain differences in individuals who may be at risk for further cognitive decline.
\end{abstract}

Key words: aging; BOLD fMRI; BOLD variability; $\mathrm{MCl}$

\section{Significance Statement}

Moment-to-moment variability in the BOLD signal, once dismissed as nuisance noise, is now understood to be an information-bearing signal. BOLD variability correlates with age and cognitive performance and was recently used to differentiate Alzheimer's disease (AD) patients from controls. As AD is a progressive disease, $A D$ patients may benefit from its early detection. We found that older adults at-risk for cognitive decline showed differences in the relationships between BOLD variability and cognitive performance, relative to healthy controls. Notably, the differences were strongest in medial temporal lobe (MTL), areas where AD is known to begin. Our data suggest that correlations between MTL BOLD variability and cognition may be useful for understanding brain differences in individuals at risk for further cognitive decline. 


\section{Introduction}

Alzheimer's disease (AD) is characterized by cognitive decline, especially to memory performance (Alzheimer's Association, 2017). Epidemiological evidence suggests age is the strongest risk factor for AD (Alzheimer's Association, 2017) and because of its progressive nature, patients may benefit from its early detection. Many studies have tried to predict $A D$ in preclinical patients (Jack and Holtzman, 2013; Jack et al., 2013; Dubois et al., 2016), but efforts are ongoing.

Temporal variability of the BOLD signal ( $\left.S D_{\mathrm{BOLD}}\right)$ correlates with age (Garrett et al., 2010) and cognitive performance (Garrett et al., 2013a, 2011). Initially, older age and poorer cognitive performance were primarily associated with lower $\mathrm{SD}_{\mathrm{BOLD}}$ during task-based $\mathrm{fMRI}$ and therefore $\mathrm{SD}_{\mathrm{BOLD}}$ was conceptualized as an indicator of brain health (Garrett et al., 2010, 2011, 2013a; Grady and Garrett, 2014; Guitart-masip et al., 2016). However, early studies of $\mathrm{SD}_{\mathrm{BOLD}}$ also found age-related increases of variability in the inferior temporal gyrus (Garrett et al., 2010), hippocampus (Garrett et al., 2011), and other areas. More recently, studies have corroborated these findings, showing aging is related to greater restingstate variability in some networks, but lower variability in others (Nomi et al., 2017). Another study found some cognitive domains were positively associated with resting-state $\mathrm{SD}_{\mathrm{BOLD}}$, whereas others were inversely related (Burzynska et al., 2015). Furthermore, in clinical populations, stroke was associated with resting-state variability increases in the left postcentral gyrus (Kielar et al., 2016); multiple sclerosis with resting-state increases in temporal gyrus and dorsal medial prefrontal cortex (Petracca et al., 2017); 22q11.2 deletion syndrome with resting-state increases in temporal lobe and caudate (Zöller et al., 2017); medial temporal lobe epilepsy with task-based increases in various cortical and subcortical regions (Protzner et al., 2013); and most notably, AD with resting-state increases in superior frontal gyrus, precentral gyrus, and putamen (Scarapicchia et al., 2018). Together, these findings raise the possibility that $\mathrm{SD}_{\mathrm{BO}} \mathrm{LD}$ may support behavior differently in healthy aging and disease.

Because of its sensitivity to age and cognitive performance, $\mathrm{SD}_{\mathrm{BO}} \mathrm{LD}$ is a promising tool for understanding age-

Received July 22, 2019; accepted February 19, 2020; First published March 13, 2020.

The authors declare no competing financial interests.

Author contributions: J.D.R. and M.D.B. designed research; T.J.G., J.V., and M.D.B. performed research; T.J.G., J.V., C.L.G., and M.D.B. analyzed data; T.J.G., J.V., J.R., C.L.G., and M.D.B. wrote the paper.

This work was supported by a Postgraduate Scholarship (doctoral) from the National Science and Engineering Research Council to T.J.G., a James S. McDonnell Scholar Award to M.D.B., a Canadian Institutes of Health Project Grant to M.D.B., a Canadian Institutes of Health Project Grant to J.D.R., and a Canadian Institutes of Health Foundation Grant to C.L.G. (MOP143311).

Correspondence should be addressed to Tyler J. Good at tgood@research. baycrest.org.

https://doi.org/10.1523/ENEURO.0290-19.2020 Copyright $(9) 2020$ Good et al.

This is an open-access article distributed under the terms of the Creative Commons Attribution 4.0 International license, which permits unrestricted use, distribution and reproduction in any medium provided that the original work is properly attributed. related pathologies like $A D$. A few studies provide hints regarding what physiological mechanisms may be driving $\mathrm{SD}_{\mathrm{BO}}$. Researchers have demonstrated a relation between age-related changes in $\mathrm{SD}_{\mathrm{BO} D}$ and dopaminergic neuromodulation (Garrett et al., 2015). Additionally, higher $\mathrm{SD}_{\mathrm{BO}}$ may reflect lower dimensional functional integration within brain networks (Garrett et al., 2018), suggesting that the optimal (and more variable) brain would have the lowest dimensionality (and therefore more tightly integrated networks) necessary given the contextual demands. This framework may explain some of the $\mathrm{SD}_{\mathrm{BOLD}}$ findings across the literature. Notably, Burzynska et al. (2015) found that resting-state $\mathrm{SD}_{\mathrm{BOLD}}$ was positively correlated with complex tasks that required greater dynamic range, but negatively associated with less complex tasks. Further, increased local variability may be common across numerous clinical populations (Protzner et al., 2013; Kielar et al., 2016; Petracca et al., 2017; Zöller et al., 2017; Scarapicchia et al., 2018) because of a shift toward suboptimal dynamics in the face of structural brain damage. Similarly, increased integration between brain networks with older age is a robust finding in healthy adults (Damoiseaux, 2017). Regardless, if SD $_{\text {BOLD }}$ reliably differs in individuals showing early signs of cognitive decline from their cognitively normal counterparts, it may prove useful in understanding the brain differences that may precede mild cognitive impairment $(\mathrm{MCl})$ and $A D$ diagnosis.

The present study compares relations between cognitive performance and $\mathrm{SD}_{\mathrm{BO} D}$ in ostensibly normal older adults identified as at-risk of developing $\mathrm{MCl}$ and agematched controls. The at-risk group scored below the threshold Montreal cognitive assessment (MoCA) score $(<26)$, whereas controls scored in the normal range. Although this grouping was based solely on MoCA score, previous investigations of this sample observed differences in other brain and behavioral domains consistent with preclinical cognitive and neural decline (Olsen et al., 2017; Yeung et al., 2017). We hypothesized that the at-risk group would have greater $\mathrm{SD}_{\mathrm{BOLD}}$ than controls, based on findings in patients with diagnosed AD (Scarapicchia et al., 2018). We also hypothesized that the relationship between $\mathrm{SD}_{\mathrm{BOLD}}$ and cognition would depend on group membership and cognitive domain. Specifically, we expected $S D_{\mathrm{BO}}$ to be positively associated with more complex tasks, whereas less complex tasks would be negatively associated with $\mathrm{SD}_{\mathrm{BOLD}}$, mirroring work by Burzynska et al. (2015). Last, as reductions in medial temporal lobe (MTL) volume were previously noted in our sample (Olsen et al., 2017; Yeung et al., 2017), we conducted a post hoc analysis to determine if $\mathrm{SD}_{\mathrm{BOLD}}$ was associated with MTL volumes in those regions showing group differences in $\mathrm{SD}_{\mathrm{BOLD}}$ identified in the present study.

\section{Methods}

\section{Participants}

Forty community-dwelling adults were recruited from participant databases at the Rotman Research Institute at 
Table 1: Demographic characteristics

\begin{tabular}{lll}
\hline & At-risk (SD) & Controls (SD) \\
$n$ & 20 & 20 \\
MoCA & $23.4(1.9)$, all $<26$ & $27.9(1.7)$, all $>26$ \\
Age & $71.5(6.5)$ & $70.3(4.5)$ \\
Education & $15.5(2.9)$ & $16.5(2.8)$ \\
Sex & 17 female & 13 female \\
\hline
\end{tabular}

Baycrest and the University of Toronto. Participants (Table 1) were chosen from the databases such that the at-risk group $(n=20)$ scored below the cutoff on the MoCA (all <26, $M=23.4, S D=1.9 ; M$ age $=71.5$, SD age $=6.1$, M education $=15.5$, SD education $=2.9,17 \mathrm{fe}-$ male), whereas the control group $(n=20)$ scored above the cutoff $(M=27.9, S D=1.7 ; M$ age $=70.3, S D$ age $=4.5$, $M$ education $=16.5$, SD education $=2.8,13$ female). There were no group differences in age, $t_{(38)}=1.3, p=0.20$, or years of education, $t_{(38)}=-2.2, p=0.40$, but a significant difference in MoCA score, $t_{(38)}=7.87, p<0.001$. The sample has been reported on, and described in more detail, in previous papers (Olsen et al., 2017; Yeung et al., 2017). No participants were removed due to head motion (absolute head motion at a single time point was no greater than $2 \mathrm{~mm}$ and relative head motion did not exceed 2.5 $\mathrm{mm}$ for all participants).

\section{Neuropsychological battery}

Participants performed a battery of neuropsychological tests to characterize their cognitive function in a separate session before the MRI scan. The battery included the Rey-Osterrieth complex figure test (RCFT; Osterreith, 1944), Visual Object and Space Perception Battery (VOSP; Warrington and James, 1991), Digit Span subset of the Wechsler Adult Intelligence Scale (WAIS; Wechsler, 1999; Wechsler et al., 2008), Logical Memory subset of the Wechsler Memory Scale (WMS), Ed 4 (Wechsler, 2009), Trail Making Test A and B (Reitan and Wolfson, 1985), and the Wechsler Abbreviated Scale of intelligence
(WASI), Ed 4 (Wechsler, 1999). Collectively, the battery assessed intelligence, visuospatial performance, cognitive control/speed, and memory (Table 2) using 14 tests.

Principal component analysis (PCA) was used ("principal" function in R) to expose the latent structure within the battery and to reduce the dimensionality of the dataset. Data were transformed if significantly skewed $(p<0.05)$ using square root, or log10 to improve the correlation structure. If necessary, variables were inverted so that higher scores always indicated better performance. Point of inflection on a scree plot was used to identify the number of components to keep. After extracting this number of factors, an oblique rotation was performed using the "principal" function in R. Factor correlations were assessed, and none exceeded 0.32 (greatest was between $\mathrm{PC} 1$ and PC4, $r=0.30$ ), indicating that $<10 \%$ of the variance was shared between factors. In this case, the solution is nearly orthogonal, and an orthogonal rotation is also appropriate (Tabachnick and Fidell, 2007). As such, a four-factor solution using a varimax rotation was performed using "principal" in R. The identified factors were largely multi-factorial and any single description would be unsatisfactory. However, as a high-level description and to allow identification at a glance, we loosely named the factors as (1) visuospatial, (2) cognitive control and speed of processing (cognitive control/speed), (3) intelligence, and (4) memory based on the primary cognitive functions assessed by the tasks that strongly contributed to each (loading $>|0.4|$; Table 2). Factor scores for each participant represent the degree to which they express the factor; higher factor scores indicate better performance on the tests reliably contributing to the factor. Factor scores were used in subsequent analyses.

\section{Imaging procedure}

All neuroimaging was performed in a single session with a 3T Siemens Trio scanner using a 12-channel head coil. Participants received a T1-weighted, magnetization-

Table 2: Factor loadings for neuropsychological tests

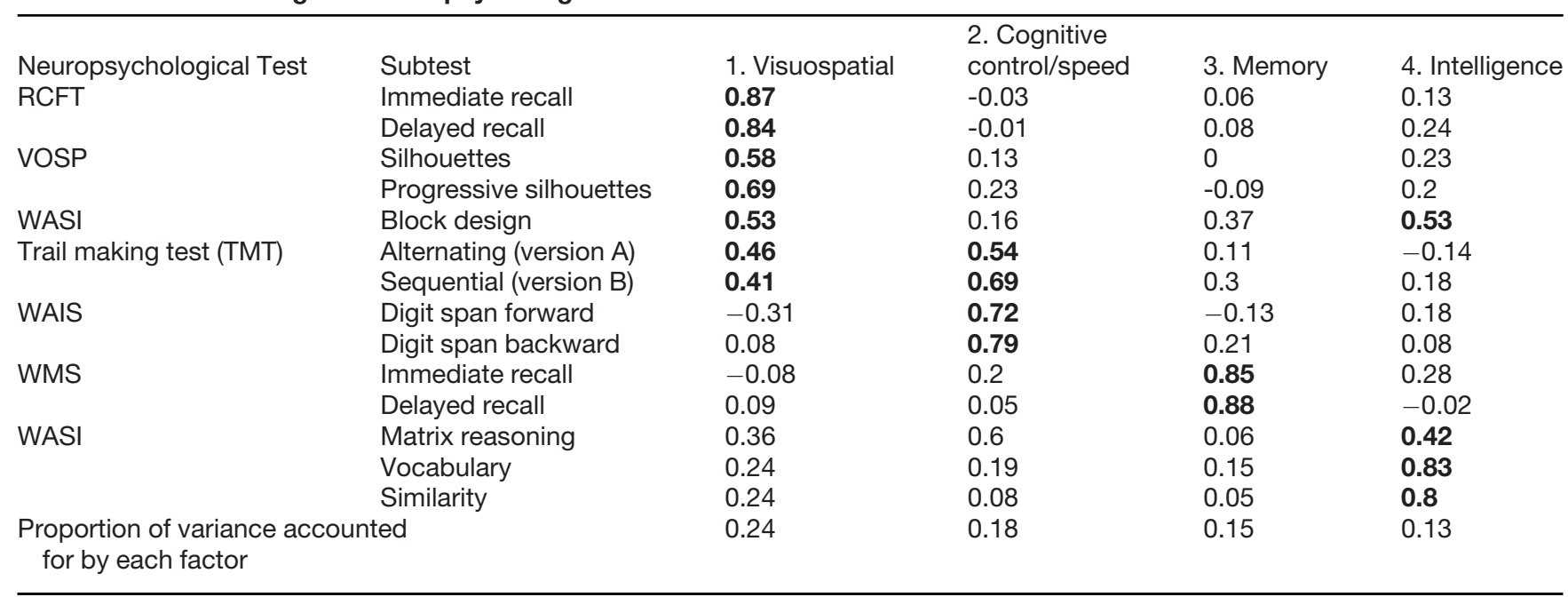

*Loadings $>|0.40|$ are bold to assist with factor interpretation. 
prepared, rapid acquisition with gradient echo image (MP-RAGE) whole-brain anatomic scan (TE/TR $=2.63 \mathrm{~ms} /$ $2000 \mathrm{~ms}, 160$ axial slices perpendicular to the AC-PC line, $256 \times 192$ acquisition matrix, voxel size $=1 \times 1 \times 1$ $\mathrm{mm}, \mathrm{FOV}=256 \mathrm{~mm}$ ). The T1-weighted MP-RAGE scan was used for slice placement during the acquisition of a subsequent high-resolution T2-weighted scan in an oblique-coronal plane, perpendicular to the hippocampal long axis (TE/TR $=68 \mathrm{~ms} / 3000 \mathrm{~ms}, 20-28$ slices depending on head size, $512 \times 512$ acquisition matrix, voxel size $=0.43 \times 0.43 \times 3 \mathrm{~mm}$, no skip, FOV $=220 \mathrm{~mm}$ ).

The DTI duration was 9:22 (min:s). There were 68 slices taken perpendicular to the AC-PC line with the following parameters: 34 directions, TE/TR $=84 \mathrm{~ms} / 7900 \mathrm{~ms}, \mathrm{FOV}=242$ $\mathrm{mm}$, flip angle $90^{\circ}, b$ value $=1000 \mathrm{~s} / \mathrm{mm}^{2}$, voxel size $=$ $2.2 \times 2.2 \times 2.2 \mathrm{~mm}$.

Participants were instructed to keep their eyes open and focused on a fixation cross during the resting-state scan. It consisted of 180 BOLD-sensitive slices $(6 \mathrm{~min}$, 180 time points, $\mathrm{TR}=2000 \mathrm{~ms}, \mathrm{TE}=30 \mathrm{~ms}$, flip angle $=$ $70^{\circ}, \mathrm{FOV}=200 \mathrm{~mm}, 30$ slices, $64 \times 64$ matrix, voxel size $=4.0 \times 4.0 \times 4.0 \mathrm{~mm})$.

\section{DTI preprocessing}

Eddy current-induced distortions were corrected using FSL's "eddy_correct" command, and the diffusion gradient vectors rotated accordingly. The MNI152_T1_1mm standard brain was then registered to subject T1-w space using a nonlinear registration conducted with Advanced Normalization Tools (ANTs). Warps produced in this step were used to transform MNI AAL cortical masks using ANTs' "WarplmageMultiTransform" executable and a nearest neighbor interpolation. FSL's FLIRT function was used to register subject $\mathrm{T} 1-w$ images to DTI space. Diffusion tensor models were fitted at each voxel by FSL's "dtifit".

\section{fMRI preprocessing}

Resting-state fMRI preprocessing was done using FMRIB's FEAT toolbox. The following steps were performed: (1) brain extraction with "bet" (Smith, 2002), (2) motion correction with MCFLIRT (Jenkinson et al., 2002), (2) slice timing correction, (3) spatial smoothing (6 mm), and (4) registration to anatomic volume using FLIRT (Jenkinson et al., 2002). Next, a bandpass filter was applied $(0.01-0.1 \mathrm{~Hz})$. Linear and quadratic detrending was performed, and then all functional volumes were examined for artefacts using independent component analysis (ICA) within-run, within-person, as implemented by FSL/ MELODIC (Beckmann and Smith, 2004). Extra de-noising using ICA was performed in light of previous research showing group differences in $\mathrm{SD}_{\mathrm{BO}}$ were enhanced following the procedure (Garrett et al., 2010). Noise components were identified using the following criteria, by two independent coders: (1) spiking (components dominated by abrupt time series spikes $\sim 26 \mathrm{SD}$ ), (2) motion (prominent edge or "ringing" effects, sometimes, but not always accompanied by large time series spikes), (3) susceptibility and flow artefacts (prominent air-tissue boundary or sinus activation, typically represents cardio/respiratory effects), (4) white matter and/or ventricle activation, (5) lowfrequency signal drift, (6) high-power in high-frequency ranges unlikely to represent neural activity $(\sim \geq 75 \%$ of total spectral power present $\sim>0.13 \mathrm{~Hz}$ ), and $(7)$ spatial distribution ["spotty", or "speckled" spatial pattern that appears scattered randomly across $\sim \geq 25 \%$ of the brain, with few if any clusters of $\sim 10$ contiguous voxels (at $4 \times$ $4 \times 4 \mathrm{~mm}$ voxel size)]. Generally, decision criteria were applied conservatively, so if there was difficulty classifying a component due to "signal" and "noise" both being present, the component was kept. Components identified as artefacts were regressed out of their respective scan using the "regfilt" function in FSL.

\section{Voxelwise diffusion tensor imaging analysis}

Voxelwise analysis of the fractional anisotropy and mean diffusivity maps was conducted using tract based spatial statistics (TBSS) with FSL's Diffusion Toolkit (Smith et al., 2006). Briefly, this involves ensuring all subjects' fractional anisotropy (FA) and mean diffusivity (MD) images were in common [Montreal Neurologic Institute (MNI)] space, using FMRIB's nonlinear registration tool, FNIRT. A group mean FA and MD image was created, and thinned to produce a mean FA/MD skeleton that represents the centers of all tracts common to the group. Each subject's FA/MD images were then projected onto this skeleton. Nonparametric permutation-based statistics were used to explore differences in FA and MD between groups using FMRIB's randomize function with 5000 unique permutations. Correction for multiple comparisons was performed using the threshold-free cluster enhancement tool in randomize with threshold $p<0.05$. Group comparison PLS could also be used to assess group differences in FA MD; however, we chose to stay within the FSL/TBSS framework, which was used to generate the FA/MD maps. To get a summary metric of overall white matter integrity, global FA and MD were calculated for each participant. These were simply the averages of all non-zero voxels in the skeletonized FA and MD images.

\section{BOLD signal variability calculation}

To calculate $\mathrm{SD}_{\mathrm{BOLD}}$, voxelwise time series were extracted from the preprocessed resting fMRI images of each subject and normalized such that the mean across the brain was 100 . The mean was then subtracted from each subject's voxelwise time series, so the data were expressed as deviation from the mean. Voxelwise $\mathrm{SD}_{\mathrm{BOLD}}$ was then calculated on these time series for each subject. We used code adapted from a previous study (https:// github.com/stefanschmidt/vartbx/; Garrett et al., 2013a), simplified to account for our data having only a single condition (resting-state). To restrict our analyses to gray matter, we masked the $\mathrm{SD}_{\mathrm{BO}}$ maps with the gray matter tissue prior provided by FSL thresholded at a probability that a given voxel is gray matter $>0.43$. We assessed head motion during the $\mathrm{fMRI}$ resting state scan and found our groups did not differ in mean absolute head motion, 
$t_{(38)}=-1.03, p=0.31$, or in mean relative head motion, $t_{(38)}=-0.23, p=0.82$.

\section{Manual segmentation of MTL subregions}

A single rater who was blind to MoCA score/group status performed manual segmentation of three hippocampal $(\mathrm{HC})$ subfields (CA1, dentate gyrus/CA2 and CA3, and subiculum) and four MTL cortices [anterolateral entorhinal cortex (alERC), posteromedial ERC (pmERC), perirhinal cortex (PRC), and parahippocampal cortex (PHC)] using the coronal slices of the T2-weighted images (in-plane resolution: $0.43 \times 0.43 \mathrm{~mm}, 3 \mathrm{~mm}$ between slices) in FSLview v3.1. A second rater who was also blind to MoCA score/group status, segmented the same regions to provide an index of inter-rater reliability. The segmentation protocol was largely similar to the Olsen-AmaralPalombo protocol used for previous volumetric investigations of the MTL (Olsen et al., 2009, 2013; Palombo et al., 2013; Yushkevich et al., 2015). The volumetric analysis of our sample has been described extensively in two previous publications (Olsen et al., 2017; Yeung et al., 2017) and therefore is not outlined further here. The at-risk group showed significantly lower alERC volume than the controls and trending lower volume in the CA1 subfield and PRC, after correction for age (Olsen et al., 2017). We also note that neither absolute nor relative head motion during the resting state scan correlated with any of the seven MTL volumes, $p>0.05$.

\section{Partial least-squares analysis}

Partial least-squares (PLS) analysis relates two sets of variables, by identifying linear combinations of variables in both sets that maximally covary together (Mclntosh and Lobaugh, 2004; Mclntosh and Mišić, 2013). In this study, we used two group comparison PLS analyses. The first related group membership to neuropsychological factor score, whereas the second related voxelwise $\mathrm{SD}_{\mathrm{BOLD}}$ to group membership. These analyses aimed to find a pattern of neuropsychological factors that differed between groups, or a pattern of brain regions where $\mathrm{SD}_{\mathrm{BOLD}}$ differed between groups. We also used behavioral PLS to find patterns of $S_{\mathrm{BO}}$. that related similarly and differently across groups to several potentially related variables (age, MoCA score, global FA/MD, and scores on our four neuropsychological factors). We included FA and MD in this analysis as a previous study found global FA was strongly associated with the relationship between neuropsychological performance and $\mathrm{SD}_{\mathrm{BOLD}}$ in a sample of healthy adults a similar age to ours (Burzynska et al., 2015). As such, we had reason to believe FA/MD may scale with $\mathrm{SD}_{\mathrm{BOLD}}$ regardless of a significant difference in white matter integrity between groups. We also used behavioral PLS to assess associations between neuropsychological test scores and age in each group. This analysis allowed use to check for differential cross-sectional changes with age in terms of cognitive performance.

For the group comparison PLS analyses, a data matrix, $\mathrm{X}$, was composed of scores on the neuropsychological factors, or a vectorized version of the $\mathrm{SD}_{\mathrm{BOLD}}$ values for all voxels within our whole-brain gray matter mask. These matrices were constructed such that observations (participants nested in groups) corresponded to rows, whereas voxels related to columns. Within-group column means were calculated, and the data in $X$ were mean-centered creating $\mathrm{M}_{\mathrm{dev}}$. The mean-centered data were then subjected to singular value decomposition (SVD): [U, S, V] = $\operatorname{SVD}\left(M_{\text {dev }}\right)$, such that USV' $=M_{\text {dev }}$. The resulting latent variables (LVs) consist of left singular vector $U$, right singular vector $\mathrm{V}$, and the diagonal matrix $\mathrm{S}$. The left singular vector $U$ contains the element saliences (weights) that identify the neuropsychological factors or voxels that make the greatest contribution to the contrast captured by the latent variable. The right singular vector $\mathrm{V}$ contains the design salience, which signifies the contribution of each group to the pattern of neuropsychological factors, or voxelwise $\mathrm{SD}_{\mathrm{BO}}$ identified by the latent variable. The scalar singular value, from the diagonal matrix $S$, denotes the covariance between the data blocks, and indicates strength of the relationship identified by the latent variable.

For the first behavioral PLS, the construction of the input matrix differed from the group comparison PLS, such that it contained the correlations between $\mathrm{SD}_{\mathrm{BO}}$ and age, MoCA score, global FA/MD, and our four cognitive factors for each group. For the second behavioral PLS, the input matrix contained correlations between age and scores on the neuropsychological tests for each group. First, correlations were calculated between the submatrices $X_{\text {nxp }}$ ( $n$ participants by $p$ voxels or $n$ participants by $p$ variables), and $Y_{\mathrm{n} \times \mathrm{b}}$ (n participants by $b$ related variables). The correlations were then stacked by group, which created the input matrix subjected to SVD. Behavioral saliences $V($ i) indicate the degree to which brain-behavior relationships are expressed for each variable in $Y$, whereas element saliences $U$ (i) indicate the degree to which voxels express these brainbehavior correlations.

Permutation testing was used to determine significance of each latent variable. Rows of the input matrix are randomly reordered and the decompositions performed, as described above. This was done 1000 times, creating a distribution of singular values. Bootstrapping was used to estimate the reliability of individual weights. Participants were randomly resampled (rows in $X$ ) with replacement while respecting group membership. The resampled matrices were decomposed, as described above. This was done 1000 times, generating a sampling distribution for the weights in the singular vectors. SE was calculated from this sampling distribution, reflecting the stability of the weight. A bootstrap ratio was then calculated for each voxel, by dividing the weight from the singular vector by its bootstrap-estimated SE, and is akin to a $z$ score. Confidence intervals were calculated around design/behavior weights using the percentiles derived from the sampling distribution. Brain scores from the behavioral PLS represent the degree to which each subject expresses the contrast identified by the latent variable.

\section{Post hoc comparison of MTL volumetry and MTL SD BOLD}

A previous study of our sample found the at-risk group had significantly reduced alERC volume relative to controls and trending lower volume in the CA1 subfield and PRC 
(Olsen et al., 2017). To assess the relation between MTL volumes and $\mathrm{SD}_{\mathrm{BOLD}}$ in these same regions, we conducted a post hoc behavioral PLS. The PLS model compared volume of three $\mathrm{HC}$ subfields (CA1, dentate gyrus/CA2 and 3, and subiculum) and four MTL cortices (alERC, pmERC, PRC, and $\mathrm{PHC}$ ) with $\mathrm{SD}_{\mathrm{BOLD}}$ in these MTL regions. Specifically, the $\mathrm{SD}_{\mathrm{BO}}$ data were calculated from all voxels in our whole-brain gray matter mask that overlapped with the left/ right hippocampal and parahippocampal regions of the automated anatomical labeling (AAL) parcellation (TzourioMazoyer et al., 2002). Critically, this included all regions quantified by volumetry. This $\mathrm{SD}_{\mathrm{BOLD}}$ data formed the first input, $X_{\text {nxp }}$, structured $n$ participants by $p$ voxels. The second input matrix, $Y_{\mathrm{nxr}}$, contained MTL volume data for each subject, $n$, by and brain region, r. Input matrices $X_{n \times p}$ and $Y_{\mathrm{nxr}}$ were subjected to behavioral PLS, as described in the previous section. Brain scores from this PLS represent the degree to which each subject expresses the contrast identified by the latent variable. Similarity of brain scores from the PLS of MTL variability and volumes and the previous behavioral PLS of whole-brain variability and potentially related variables was assessed with Pearson correlation. This showed the degree of association between the pattern of $\mathrm{SD}_{\mathrm{BOLD}}$ related to the behavioral variables and the pattern of $\mathrm{SD}_{\mathrm{BOLD}}$ related to $\mathrm{MTL}$ volumes.

\section{Results}

\section{No group differences on brain variables, cognitive measures}

Before probing the relations among our variables, we checked for differences in $\mathrm{SD}_{\mathrm{BO}}$, $\mathrm{FA}$, and $\mathrm{MD}$ between the at-risk group and controls. A group comparison PLS revealed no significant difference in voxelwise wholebrain gray matter $\mathrm{SD}_{\mathrm{BOLD}}$ between those at-risk for $\mathrm{MCl}$ and controls $(p>0.05)$. Non-parametric permutationbased testing using FSL's "randomise" found no significant group differences in voxelwise FA or MD $(p>0.05)$. Similar to this finding with randomize, group comparison PLS of voxelwise FA and MD showed no group differences (FA, $p=0.68$; MD, $p=0.17$ ).

Our PCA of the neuropsychological battery revealed four significant factors, which we designated as follows: (1) visuospatial, (2) cognitive control/speed, (3) memory, and (4) intelligence (Table 2). The groups did not significantly differ in their performance on any of the factors; however, a group comparison PLS showed the at-risk group trended toward lower scores on the control/speed, memory, and intelligence factors $(p=0.053)$. We also note that the visuospatial factor correlated moderately with age, $r_{(38)}=-0.36, p=0.02$; Fig. 1, though the other factors did not $(p>0.05)$. For group differences on the individual neuropsychological tests please refer to Table 2 in an earlier paper: Olsen et al., 2017. Briefly, the at-risk group had significantly lower WMS recognition accuracy and WASI matrix reasoning scores $(p<0.01)$, lower scores on the WMS delayed recall, digit span backward, Trails B, VOSP incomplete letters and number locations $(p<0.05)$, and trending lower scores on the digit span forward, WASI vocabulary, WASI similarity, and VOSP position discrimination $(p<0.1)$. A behavioral PLS (Fig. 2) found age was associated with scores on the VOSP silhouette, VOSP progressive silhouette, and WASI matrix reasoning tests in the at-risk group, but not controls.

\section{$\mathrm{SD}_{\mathrm{BOLD}}$ correlates with demographic, brain structure, and cognitive variables}

The omnibus behavioral PLS compared the relations between voxelwise $S_{B}$ BOLD and several potentially related variables (age, MoCA score, global FA, global MD, and scores on our 4 cognitive factors) in both groups. A single significant latent variable was identified, $p<0.001,41.1 \%$ covariance. This LV (Fig. 3) showed that in controls, higher $\mathrm{SD}_{\mathrm{BOLD}}$ was related to higher scores on the cognitive control/speed and intelligence factors, whereas lower $\mathrm{SD}_{\mathrm{BOLD}}$ was related to greater global MD and higher scores on the visuospatial and memory factors. The voxels that most reliably related to this LV were located in the posterior medial temporal lobes, hippocampus, visual cortex, and striatum (Fig. 3B; Table 3). In the at-risk group, we observed the same pattern as in controls of lower $\mathrm{SD}_{\mathrm{BO}}$ related to higher scores on the visuospatial factor. However, higher $\mathrm{SD}_{\mathrm{BO}}$ was also related to older age and lower scores on the control/speed factor in the at-risk group. That is, the positive relation between the cognitive control/speed factor and $\mathrm{SD}_{\mathrm{BOLD}}$ seen in the control group was reversed in the at-risk group. Notably, there was no relation between age and $\mathrm{SD}_{\mathrm{BOLD}}$ in controls and no relation between memory and intelligence and $\mathrm{SD}_{\mathrm{BO}}$ in the at-risk group.

\section{MTL SD BOLD $_{\text {correlates with MTL volumes }}$}

An omnibus behavioral PLS compared bilateral MTL $\mathrm{SD}_{\mathrm{BOLD}}$ with gray matter volume of three hippocampal subfields and four MTL cortices in both groups (Fig. 4A, $B)$. A single significant LV ( $<<0.0001,72.0 \%$ covariance) showed that reduced volume was associated with greater MTL $S D_{\text {BOLD, }}$ especially in the at-risk group. Specifically, reduced volumes in the CA1, CA3/DG, PHC, and alERC were related to higher MTL $S D_{B O L D}$ in the at-risk group. In the control group, the same pattern of greater MTL $\mathrm{SD}_{\mathrm{BOLD}}$ was associated with lower $\mathrm{PHC}$ volume.

Brain scores from the PLS of MTL variability and volume were highly correlated with brain scores from the wholebrain variability and behavioral PLS in the control $(r=0.91$, $p<0.0001)$ and at-risk $(r=0.89, p<0.0001)$ groups (Fig. $4 C$ ). This suggests the pattern of $\mathrm{SD}_{\mathrm{BO}}$ as associated with behavioral performance is highly correlated with the pattern of $S_{\text {BOLD }}$ related to MTL volumes. Brain scores from the PLS comparing MTL variability and volumes (Fig. $4 A$, $B$ ) were not correlated with memory factor scores in both groups (at-risk: $r=0.13, p=0.57$; controls: $r=-0.39$, $p=0.09$ ).

\section{Discussion}

We investigated the relations between BOLD signal variability and cognitive performance in a group of healthy older adults and a group of older adults at-risk for 

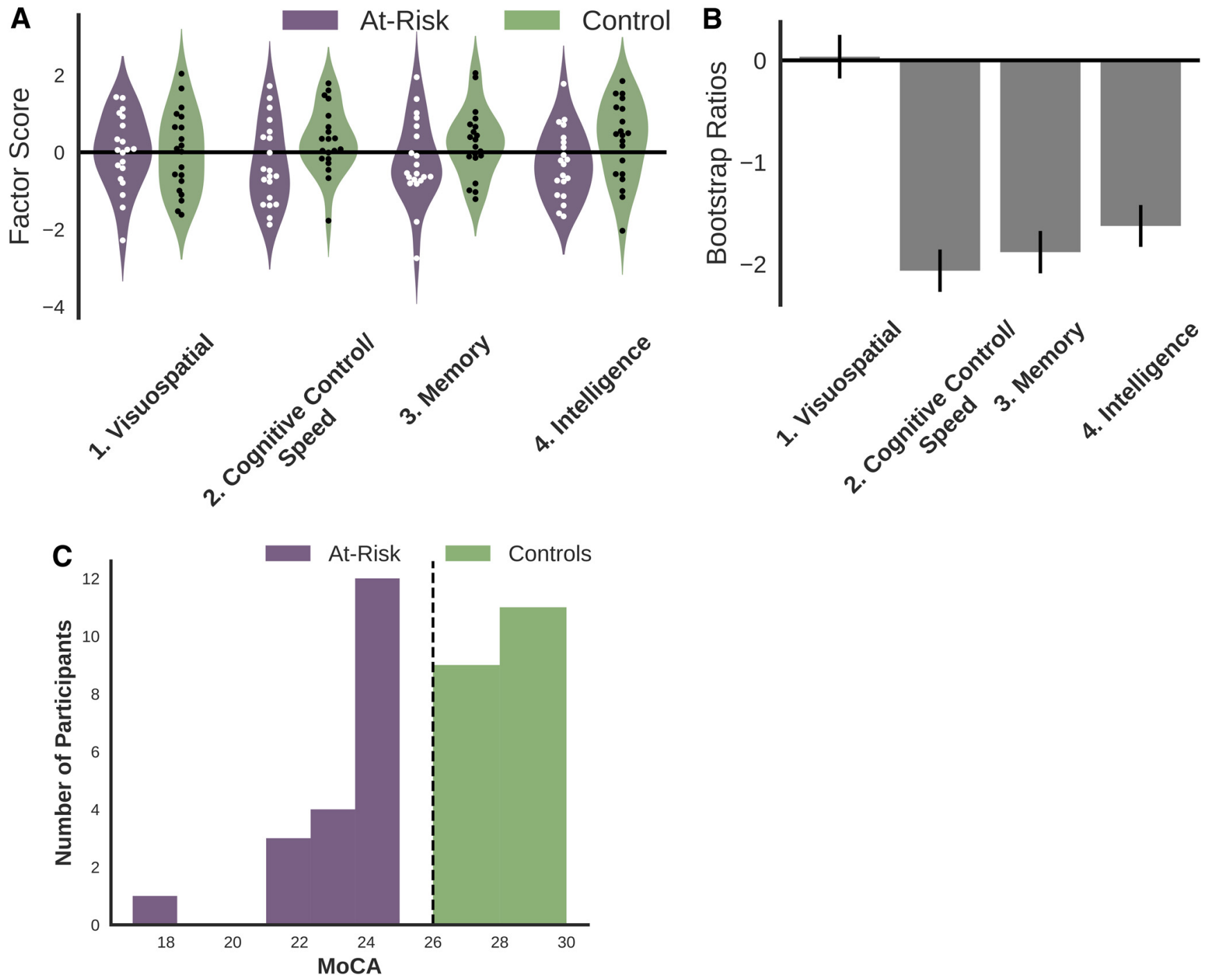

Figure 1. The at-risk group scored lower than controls on three neuropsychological factors (cognitive control/speed, memory, and intelligence), though the group difference did not quite reach significance. $\boldsymbol{A}$, Violin plots showing the distribution of factor scores for each group. $\boldsymbol{B}$, Results from a group comparison PLS that found a marginal effect such that the at-risk group scored lower than controls on the cognitive control, memory and intelligence factors $(p=0.053)$. Bootstrap ratios (BSRs) are plotted and represent a linear combination of the factors weighted by how strongly they contribute to the latent variable. Negative BSRs indicate the at-risk group had lower scores than controls on these factors. BSRs may be interpreted similar to $z$ score $(>|2.5|$ akin to $p<0.05)$, suggesting again that the at-risk group's lower scores on the control/speed, memory, and intelligence factors was approaching significance. Error bars represent $1 \mathrm{SE}$ = standard error. $\mathbf{C}$, Distribution of MoCA scores in the present sample. The black dashed line indicates the recommended MoCA cutoff score (26 points out of 30 ).

cognitive decline, as documented by their sub-threshold MoCA score and reduced MTL volumes (Olsen et al., 2017). Our study demarcated older adults into at-risk and control groups based on MoCA score because the MoCA has demonstrated association with cognitive performance (D'Angelo et al., 2016; Yeung et al., 2017; Ryan et al., 2019), electrophysiological signature (Newsome et al., 2013), MTL volume (Olsen et al., 2017), strong sensitivity and specificity for $\mathrm{MCl}$ (Nasreddine et al., 2005; Markwick et al., 2012), and prediction of future conversion to $A D$ (Julayanont et al., 2014). In healthy older adults greater $\mathrm{SD}_{\mathrm{BOLD}}$ was associated with higher cognitive control/ speed and intelligence scores, but lower memory and visuospatial scores. Notably, this effect was not as robust in the at-risk group. The at-risk group demonstrated neither the positive correlations observed between $\mathrm{SD}_{\mathrm{BOLD}}$, cognitive control/speed and intelligence, nor the negative correlation between $\mathrm{SD}_{\mathrm{BOLD}}$ and memory scores. The effect observed in controls was most robust in the MTL, where AD pathology is known to begin (Braak and Braak, 1991; Braak et al., 1993) and where our sample of at-risk individuals had significantly reduced volume (Olsen et al., 2017). We found that greater $S D_{\text {BOLD }}$ in the MTL was correlated with lower parahippocampal volume in both groups, but that this relation involved more MTL regions in the at-risk group. Critically, we also found no group 

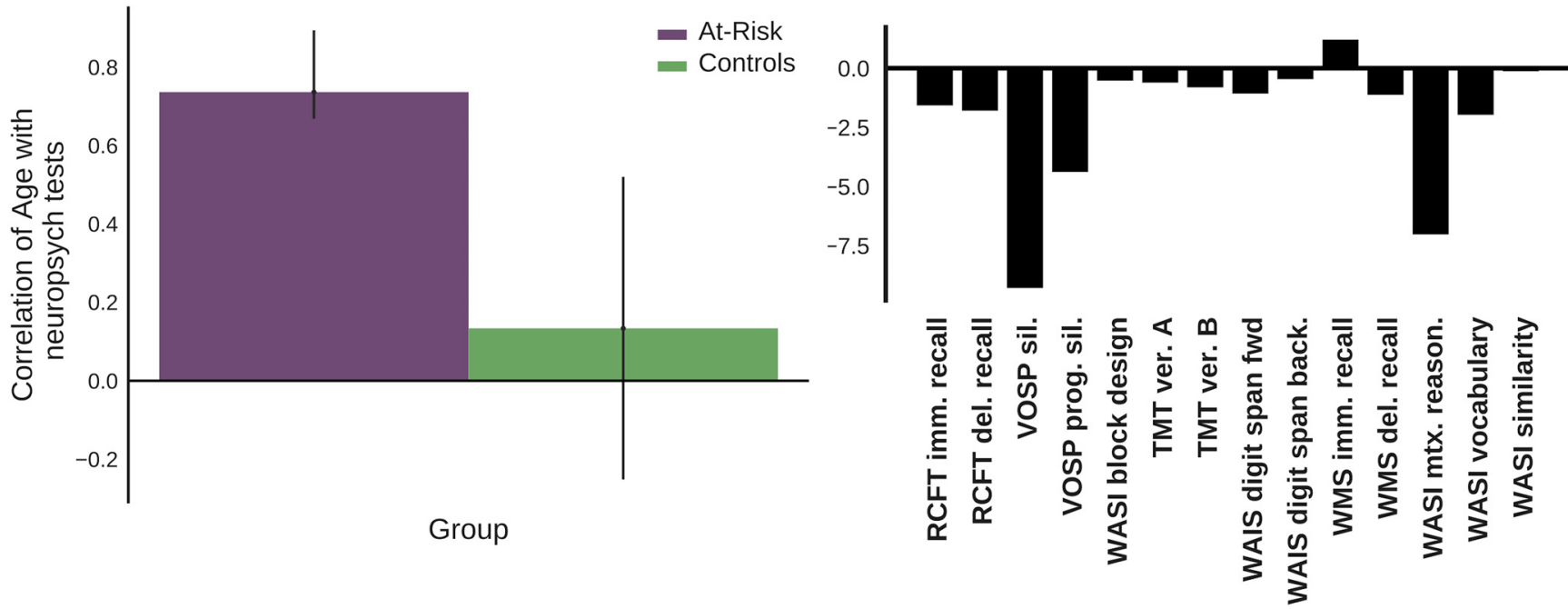

Figure 2. A behavioral PLS ( $p=0.01,87 \%$ covariance) found age was negatively correlated with scores on the VOSP silhouette, VOSP progressive silhouette, and WASI matrix reasoning tests in the at-risk group, but not controls. The left bar graph shows the correlation between age and the pattern of neuropsychological tests shown on the right. The error bars represent $95 \%$ confidence intervals. Bootstrap ratios are pictured on the right, representing a linear combination of the neuropsychological tests weighted by how much they contribute to the latent variable. Bootstrap ratios may be interpreted like $z$ scores, such that bootstrap ratios $>|2.5|$ are considered to be reliably correlated with age in the group(s) that are significantly contributing to the latent variable. Immediate (imm), delayed (del), sillhoutte (sil), version (Ver).

difference in $\mathrm{SD}_{\mathrm{BOLD}}$ between groups. Together, our results suggest that cortical atrophy may lead to greater brain signal variability. Moreover, these changes may explain the differential relations observed between brain signal variability and cognitive control/speed performance in both groups. In the following we discuss implications for the role of $\mathrm{SD}_{\mathrm{BOLD}}$ in cognition of healthy older adults and in those at-risk for developing $\mathrm{MCl}$.
A

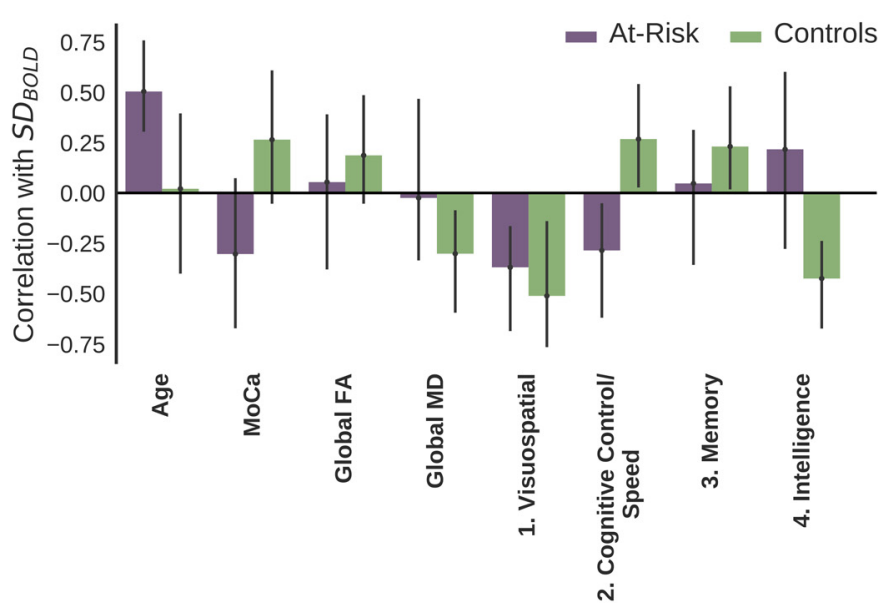

B

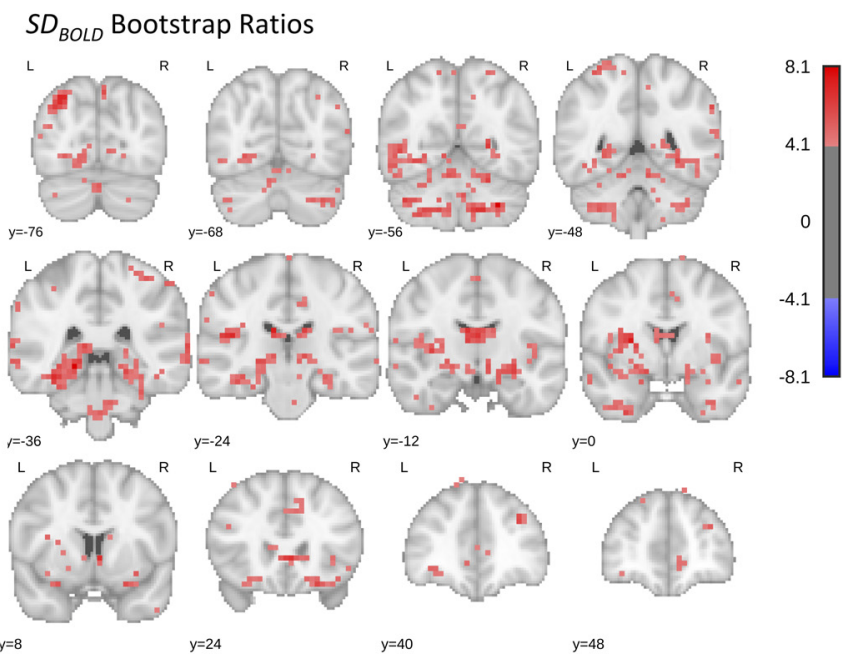

Figure 3. Multivariate PLS analysis of the relationship between $\mathrm{SD}_{\mathrm{BOLD}}$ and 8 variables (age, MoCA score, global FA and MD, and score on 4 neuropsychological factors). In controls, $\mathrm{SD}_{\mathrm{BOLD}}$ (particularly in the MTL) was associated with higher cognitive control/ speed and intelligence scores, but lower visuospatial and memory scores. These relationships were weaker in the at-risk group. $\boldsymbol{A}$, The first latent variable $(p<0.001,41.1 \%$ covariance) from the omnibus, between groups behavioral PLS assessing correlations between the 8 variables and $S_{B} D_{B O L D}$. The bars represent the correlation between each variable with the pattern of $S_{B O L D}$ shown in the corresponding brain plot $(\boldsymbol{B})$. The error bars represent $95 \%$ confidence intervals, so the error bars of variables significantly contributing to the latent variable will not cross zero. B Brain plots showing the bootstrap ratios for the latent variable, which may be interpreted like $z$ scores. That is, the highlighted voxels are reliably associated with the related variables in $A$ that significantly contribute to the latent variable. To clearly show the spatial pattern of the respective latent variable, only voxels with bootstrap ratios $>|4|$ are pictured. 
Table 3. Significant clusters representing relationship between $\mathrm{SD}_{\mathrm{BO}}$ and eight variables (Fig. 3)

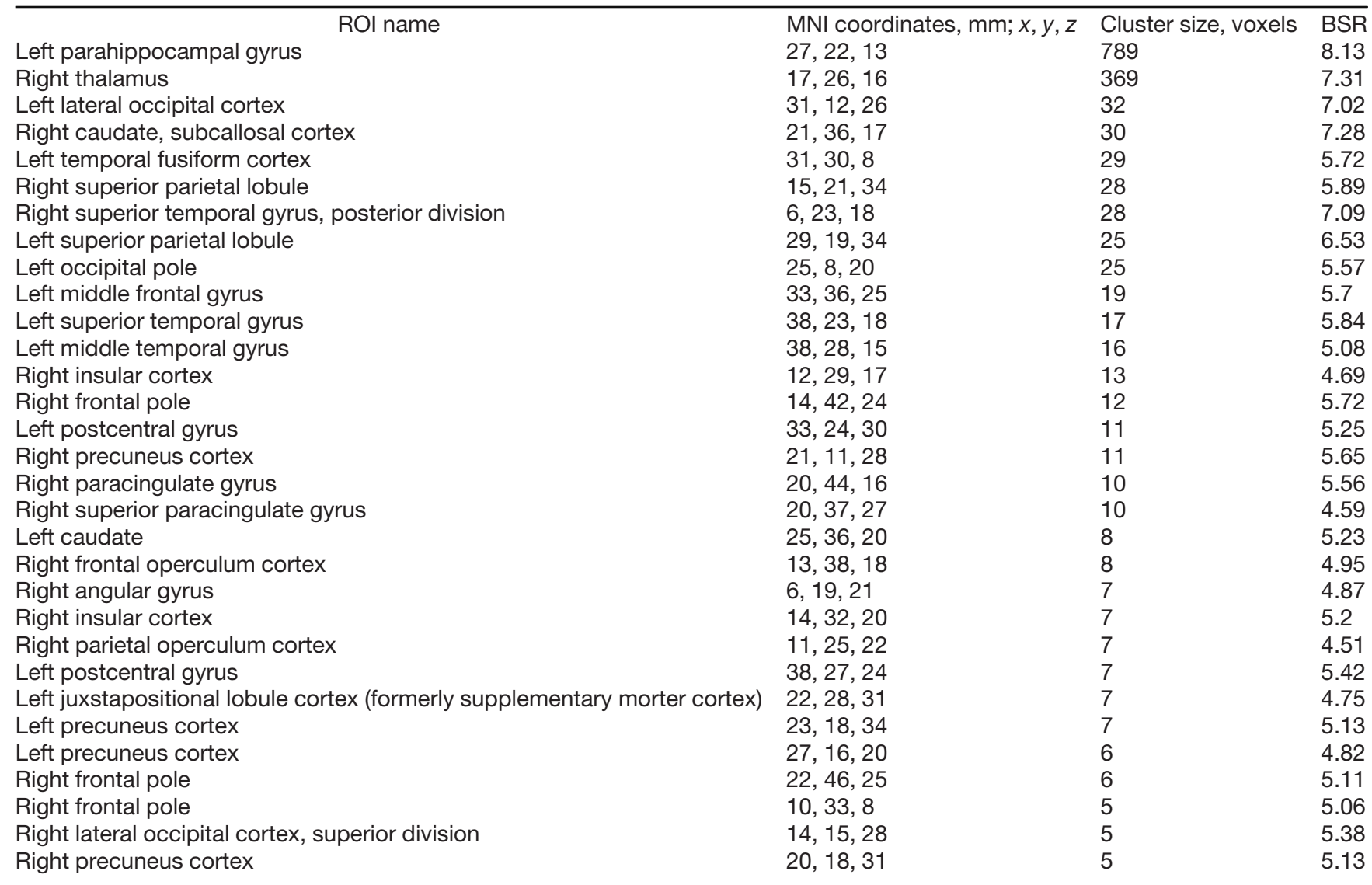

Only clusters of five voxels or larger with bootstrap ratio (BSR) $>|4|$ are included.

Our first analyses assessed correlations between $\mathrm{SD}_{\mathrm{BOLD}}$ and several potentially related variables: age, MoCA score, global FA, global MD, and scores on our four cognitive factors. In controls, we found that greater BOLD signal variability, particularly in posterior MTL regions, was related to lower global MD, suggesting generally greater white matter integrity. In terms of cognition, greater BOLD signal variability was related to better performance on the cognitive control/speed and intelligence factors, but poorer performance on the visuospatial and memory factors. Our results corroborated previous work, which found that the direction of the relationship between $\mathrm{SD}_{\mathrm{BOLD}}$ and cognitive factors was dependent on cognitive domain and correlated with global white matter integrity in healthy older adults (Burzynska et al., 2015). The cognitive control/speed and intelligence factors may have required greater dynamic range than visuospatial and memory factors, driving the different relations with $\mathrm{SD}_{\mathrm{BO}}$, as first hypothesized by Burzynska et al., 2015.

The regions where we observed a correlation between $\mathrm{SD}_{\mathrm{BOLD}}$ and cognition have been associated with various cognitive functions. Briefly, the bilateral superior parietal regions are primarily associated with voluntary control of attention during perception, but may also be involved with memory retrieval, monitoring, and verification (Cabeza et al., 2008). The hippocampus is broadly understood to support episodic memory (Moscovitch et al., 2016). The thalamus relays sensory and motor signals to the cerebral cortex and is implicated in consciousness and sleep (Steriade and Llinás, 1988). The fusiform and inferior temporal cortex are involved in the ventral visual stream and shape processing (Haxby et al., 1991; Denys et al., 2004). Last, the insular cortex has multiple functions including multimodal sensory processing and binding (Bushara et al., 2003) and a role in emotion (Phan et al., 2002). A similarity among many of these regions is that they have been identified as network hubs (van den Heuvel and Sporns, 2011). Hubs are identified as well-connected regions through structural or functional network analyses. As was initially proposed by Burzynska et al. (2015), we suggest that the high connectivity of these regions may result in or require greater neural variability, which would be reflected by greater $\mathrm{SD}_{\mathrm{BOLD}}$. That is, greater $\mathrm{SD}_{\mathrm{BOLD}}$ in these regions might indicate more flexible network integration, which presumably would support higher scores on the cognitive control/speed and intelligence factors.

We report a negative association between $M T L S_{B O L D}$ and our memory factor, whereas Burzynska et al. (2015) reported a positive relationship and other studies have shown $\mathrm{SD}_{\mathrm{BO}}$ LD supports other types of cognitive function (Garrett et al., 2011, 2013a). However, another recent study found a negative association between $\mathrm{SD}_{\mathrm{BO}}$ and memory scores, though this was in a sample of AD patients (Scarapicchia et al., 2018). Regardless, the 
A

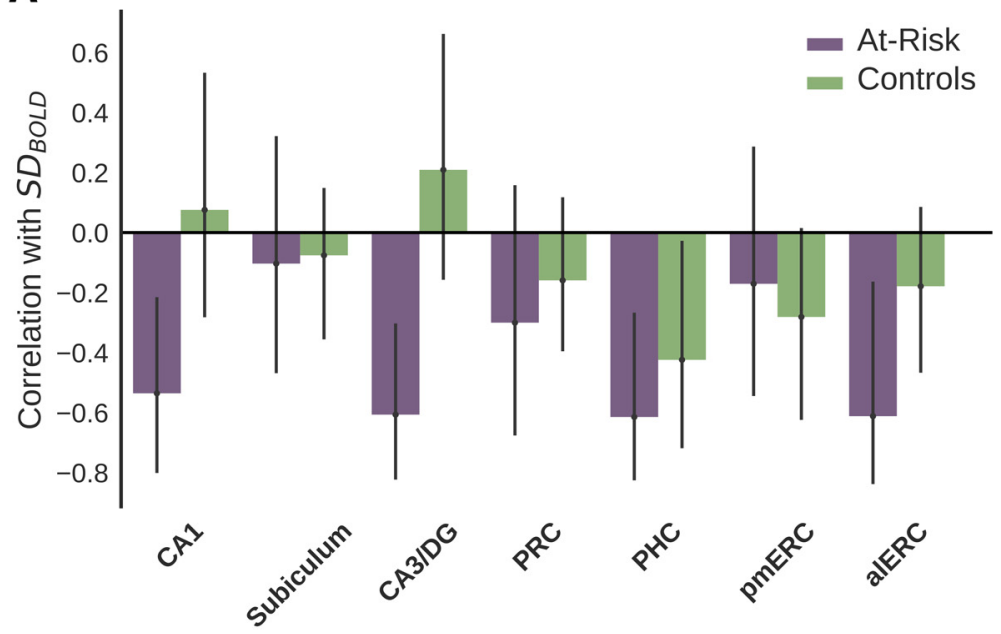

B

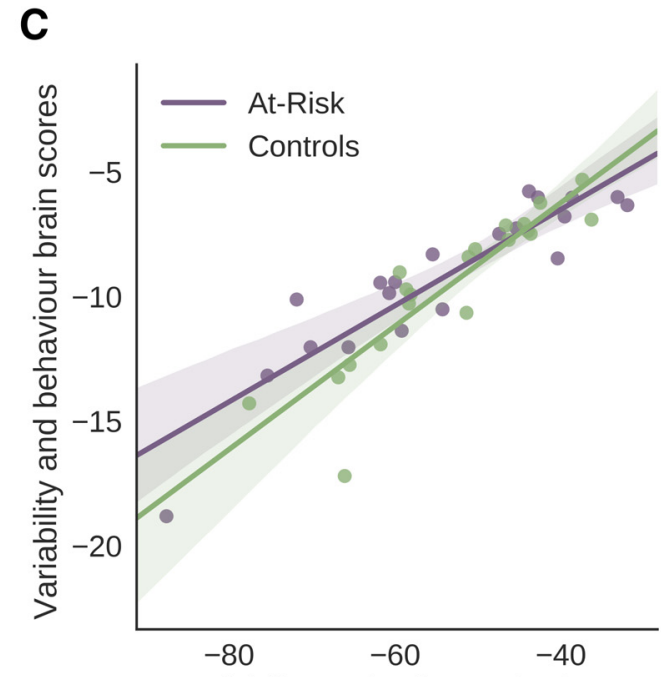

MTL variability and volumes brain scores

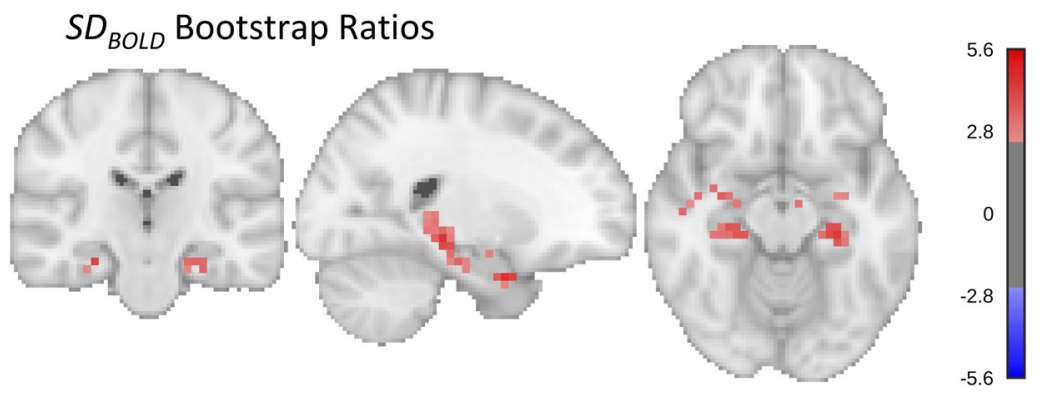

Figure 4. Multivariate PLS analysis of the relationship between $\mathrm{SD}_{\mathrm{BOLD}}$ and gray matter volume in the $\mathrm{HC}$ and $\mathrm{MTL}$. In the at-risk group, reduced volumetry in the CA1, CA3/DG, PHC, and alERC was associated with higher $\mathrm{SD}_{\mathrm{BO}}$ in the MTL. The effect was less widespread in controls. $\boldsymbol{A}, \boldsymbol{B}$, The first latent variable $(p<0.0001,72.0 \%$ covariance) from the omnibus between groups behavioral PLS assessing correlations between volumetry of 7 MTL regions (3 hippocampal subfields and 4 MTL subregions) and MTL $\mathrm{SD}_{\mathrm{BOLD}}$. $\boldsymbol{A}$, The bars represent the correlation between each MTL subregion with the pattern of $\mathrm{SD}_{\mathrm{BOLD}}$ shown in the corresponding brain plot $(\boldsymbol{B})$. The error bars represent $95 \%$ confidence intervals, so the error bars of variables significantly contributing to the latent variable will not cross zero. $\boldsymbol{B}$, Brain plots showing the bootstrap ratios for the latent variable, which may be interpreted like $\boldsymbol{Z}$ scores. Voxels with bootstrap ratios $>|2.5|$ are pictured and considered to be reliably correlated with the brain volumes that are significantly contributing to the latent variable. $\boldsymbol{C}$, Scatter plot shows the correlation between brain scores from the PLS comparing whole-brain $\mathrm{SD}_{\mathrm{BOLD}}$ and several predictor variables (Fig. 3) and the brain scores from the PLS comparing MTL variability and volumes. The strong correlation (at-risk: $r=0.89, p<0.0001$; controls: $r=0.91, p<0.0001$ ) indicates that the pattern of $\mathrm{SD}_{\mathrm{BOLD}}$ that is associated with behavior is highly similar to the pattern of $\mathrm{SD}_{\mathrm{BOLD}}$ associated with MTL volumes. Brain volumes: HC subfields (CA1, dentate gyrus/CA2 and 3, and subiculum) and MTL cortices (alERC, pmERC, PRC, and PHC).

discrepancy highlights the need for further study with larger samples and standardized methodology. We also note the possibility that differences in the neuropsychological battery and preprocessing choices may contribute to the observed inconsistencies across the literature. The memory factors of the present study, Scarapicchia et al. (2018) and Burzynska et al. (2015) were composed of different tests, which require use of different cognitive constructs to varying degrees (e.g., in the use of targets that tax verbal vs visuospatial ability). Additionally, we note that the present study and Burzynska et al. (2015) denoised the fMRI data, but Scarapicchia et al. (2018) did not, and prior work has shown the importance of removing as much unwanted noise as possible when examining SD $_{\text {BOLD }}$ (Garrett et al., 2010; Grady and Garrett, 2014).
Our initial omnibus PLS found that older age, and lower scores on the visuospatial and cognitive control/speed factors were related to greater BOLD signal variability in the at-risk group. The positive relationship between visuospatial performance and $\mathrm{SD}_{\mathrm{BOLD}}$ was common in the atrisk and control groups. In the at-risk group however, $\mathrm{SD}_{\mathrm{BOLD}}$ also correlated positively with age. Earlier work observed that $\mathrm{SD}_{\mathrm{BOLD}}$ in $\mathrm{fMRI}$ task-based contexts was reduced in older age (Garrett et al., 2011, 2013b; Grady and Garrett, 2014, 2018). However, as we observed here, some of these studies found isolated brain regions with increased $\mathrm{SD}_{\mathrm{BOLD}}$ during rest and task-fMRI associated with age (Garrett et al., 2010, 2011; Nomi et al., 2017). Most notably, Garrett et al. (2010) found age-related increases in the MTL, paralleling our finding in the at-risk group. Therefore, although this relation between $\mathrm{SD}_{\mathrm{BOLD}}$ 
and age was somewhat weak in the at-risk group, the effect was nevertheless consistent with prior work. The group difference in age- $\mathrm{SD}_{\mathrm{BOLD}}$ correlation was unexpected, but likely reflects greater age-related variation in the neuropsychological battery (Fig. 2) in the at-risk group, rather than difference in $\mathrm{SD}_{\mathrm{BO}}$. We advocate multivariate techniques such as PLS and canonical correlation, which can unmask rich relations between age, other demographic variables, and brain function (Ferreira et al., 2016; Perry et al., 2017).

Critically, we found the at-risk group did not show the positive correlations observed in controls between intelligence, cognitive control/speed, and $\mathrm{SD}_{\text {BOLD. Whereas }}$ controls showed a positive relationship between control/ speed and $\mathrm{SD}_{\mathrm{BOLD}}$, the at-risk group showed a negative relationship. Similarly, controls showed a positive relationship between intelligence and $\mathrm{SD}_{\mathrm{BO}}$, but this relation was non-significant in the at-risk group. Additionally, we found a negative relationship in controls between memory performance and $\mathrm{SD}_{\mathrm{BO}}$, whereas this effect was absent in the at-risk group. Our results suggest that alterations in the relations between cognitive performance and $\mathrm{SD}_{\mathrm{BOLD}}$ may occur in individuals at-risk for further cognitive decline, including $\mathrm{MCl}$. Importantly, the effect was most robust in the MTL, where $A D$ pathology is known to begin (Braak and Braak, 1991; Braak et al., 1993), and where this sample of ostensibly healthy older adults showed reduction in gray matter volume (Olsen et al., 2017).

In both groups, lower parahippocampal volume was associated with greater $\mathrm{SD}_{\mathrm{BOLD}}$ in MTL brain areas. Interestingly, the effect was more widespread in the atrisk group, where lower volume of the CA1, CA3/DG, and alERC were also related to greater $\mathrm{SD}_{\mathrm{BO}} \mathrm{LD}$. Importantly, a previous study with our sample found that reduced volume in the MTL, especially the alERC, CA1/DG, and PRC were strongly associated with MoCA performance (Olsen et al., 2017). Furthermore, volume in the alERC has been linked to object processing in this sample (Yeung et al., 2017). Together, these results suggest lower MTL volume is likely related to greater $S_{B O L D}$ in the MTLs of older adults regardless of cognitive status; however, the relationship was more reliable in the at-risk group, possibly because of greater variability in MTL subregion volumes. Furthermore, the correlation between brain scores from both PLS analyses (Figs. 3. 4), suggests a strong similarity between the patterns of $S_{\text {BOLD }}$ associated with MTL volumes and behavioral performance. In fact, we found a trend for a similar negative correlation between the brain scores from the MTL variability and volumes PLS and the memory factor in controls, but not the at-risk group, similar to what was observed in the $\mathrm{SD}_{\mathrm{BOLD}}$ and behavior PLS (Fig. 4). Given the exclusively negative associations between $\mathrm{SD}_{\mathrm{BOLD}}$ and cognitive performance in the at-risk group, greater variability may represent a failed attempt to compensate for cortical atrophy in the MTL. In contrast, the controls showed positive associations between $\mathrm{SD}_{\mathrm{BOLD}}$ and the cognitive control/speed and intelligence factors, possibly because they have better maintained MTL volumes.
Despite finding robust group differences in the relations between $\mathrm{SD}_{\mathrm{BO}}$ and our demographic and behavioral variables across groups, we did not observe a group difference in $\mathrm{SD}_{\mathrm{BO}}$. Patients with various clinical diagnoses including stroke (Kielar et al., 2016), multiple sclerosis (Petracca et al., 2017), 22q11.2 deletion syndrome (Zöller et al., 2017), and AD (Scarapicchia et al., 2018) have demonstrated greater $\mathrm{SD}_{\mathrm{BOLD}}$ relative to comparison groups. However, our sample of at-risk participants, who presented with only early warning signs of cognitive decline that did not meet diagnostic criteria for $\mathrm{MCl}$, did not show a difference in $\mathrm{SD}_{\mathrm{BOLD}}$. We acknowledge $\mathrm{SD}_{\mathrm{BOLD}}$ is sensitive to decisions made when preprocessing the raw data (Garrett et al., 2010; Turner et al., 2015). To this end, we highlight the careful de-noising of our data, which we view as an important step for analyses of $\mathrm{SD}_{\mathrm{BOLD}}$, as it has been shown to enhance age effects (Garrett et al., 2010) and should function to reduce the influence of "junk" noise. If de-noising enhances group differences then we can have confidence in our finding of no group differences in $\mathrm{SD}_{\mathrm{BOLD}}$ per se, as de-noising should have made it easier to such differences if they existed.

We acknowledge the cognitive factors used this study require contribution from multiple cognitive domains. As such, future studies should aim to pinpoint the relations between specific cognitive functions and $\mathrm{SD}_{\mathrm{BO}}$ and how these associations may change in disease. We also recognize our small sample size $(N=40)$ limits the generalizability of our findings, and highlight the need for longitudinal studies that follow the "at-risk" group. Last, variability of resting-state $\mathrm{SD}_{\mathrm{BOLD}}$ is contentious, particularly in the context of aging (Tsvetanov et al., 2015, 2019). In this regard, we note that our study did not focus exclusively on age effects and again highlight our careful denoising of the data, which addresses artifact from cardiovascular and neurovascular signal.

\section{Conclusions}

We found that a group of ostensibly healthy older adults previously identified as at-risk (Olsen et al., 2017) showed a different relation between $\mathrm{SD}_{\mathrm{BO}} \mathrm{LD}$ and cognition than did controls. In controls, $\mathrm{SD}_{\mathrm{BO}} \mathrm{SD}$ was positively correlated with tasks of cognitive control/speed and intelligence, but negatively correlated with memory and visuospatial scores. In contrast, these relations were weaker in the at-risk group. Notably, the relations between $\mathrm{SD}_{\mathrm{BO}}$ and and cognition observed in controls were most robust in MTL regions, where AD pathology first occurs (Braak and Braak, 1991; Braak et al., 1993). In addition, we showed that both groups had a negative relation between parahippocampal volume and MTL $\mathrm{SD}_{\mathrm{BO}}$, however, the effect was more widespread in the at-risk group. Our findings provide evidence that brain signal variability may increase in the face of cortical atrophy, leading to the differential relations observed between brain signal variability and cognitive performance in groups of different cognitive status.

\section{References}

Alzheimer's Association (2017) 2017 Alzheimer's disease facts and figures. Alzheimers Dement 13:325-373. 
Beckmann CF, Smith SM (2004) Probabilistic independent component analysis for functional magnetic resonance imaging. IEEE Trans Med Imaging 23:137-152.

Braak H, Braak E (1991) Neuropathological stageing of Alzheimer-related changes. Acta Neuropathol 82:239-259.

Braak H, Braak E, Bohl J (1993) Staging of Alzheimer related cortical destruction. Eur Neurol 33:403-408.

Burzynska AZ, Wong CN, Voss MW, Cooke GE, McAuley E, Kramer AF (2015) White matter integrity supports BOLD signal variability and cognitive performance in the aging human brain. PLoS One 10:e0120315-17.

Bushara KO, Hanakawa T, Immisch I, Toma K, Kansaku K, Hallett M (2003) Neural correlates of cross-modal binding. Nat Neurosci 6:190-195.

Cabeza R, Ciaramelli E, Olson IR, Moscovitch M (2008) Parietal cortex and episodic memory: an attentional account. Nat Rev Neurosci 9:613-625.

Damoiseaux JS (2017) Effects of aging on functional and structural brain connectivity. Neuroimage 160:32-40.

D'Angelo MC, Smith VM, Kacollja A, Zhang F, Binns MA, Barense MD, Ryan JD (2016) The effectiveness of unitization in mitigating age-related relational learning impairments depends on existing cognitive status. Neuropsychol Dev Cogn B Aging Neuropsychol Cogn 23:667-690.

Denys K, Vanduffel W, Fize D, Nelissen K, Peuskens H, Van Essen D, Orban GA (2004) The processing of visual shape in the cerebral cortex of human and nonhuman primates: a functional magnetic resonance imaging study. J Neurosci 24:2551-2565.

Dubois B, Hampel H, Feldman HH, Scheltens P, Aisen P, Andrieu S, Bakardjian H, Benali H, Bertram L, Blennow K, Broich K, Cavedo E, Crutch S, Dartigues JF, Duyckaerts C, Epelbaum S, Frisoni GB, Gauthier S, Genthon R, Gouw AA (2016) Preclinical Alzheimer's disease: definition, natural history, and diagnostic criteria. Alzheimers Dement 12:292-323.

Ferreira LK, Regina ACB, Kovacevic N, da Graça Morais Martin M, Santos PP, Carneiro C d G, Kerr DS, Amaro E, McIntosh AR, Busatto GF (2016) Aging effects on whole-brain functional connectivity in adults free of cognitive and psychiatric disorders. Cereb Cortex 26:3851-3865.

Garrett DD, Kovacevic N, McIntosh AR, Grady CL (2010) Blood oxygen level-dependent signal variability is more than just noise. J Neurosci 30:4914-4921.

Garrett DD, Kovacevic N, McIntosh AR, Grady CL (2011) The importance of being variable. J Neurosci 31:4496-4503.

Garrett DD, Kovacevic N, McIntosh AR, Grady CL (2013a) The modulation of BOLD variability between cognitive states varies by age and processing speed. Cereb Cortex 23:684-693.

Garrett DD, Samanez-Larkin GR, MacDonald SWS, Lindenberger U, McIntosh AR, Grady CL (2013b) Moment-to-moment brain signal variability: a next frontier in human brain mapping? Neurosci Biobehav Rev 37:610-624.

Garrett DD, Nagel IE, Preuschhof C, Burzynska AZ, Marchner J, Wiegert S, Jungehülsing GJ, Nyberg L, Villringer A, Li SC, Heekeren HR, Bäckman L, Lindenberger U (2015) Amphetamine modulates brain signal variability and working memory in younger and older adults. Proc Natl Acad Sci U S A 112:7593-7598.

Garrett DD, Epp SM, Perry A, Lindenberger U (2018) Local temporal variability reflects functional integration in the human brain. Neuroimage 183:776-787.

Grady CL, Garrett DD (2014) Understanding variability in the BOLD signal and why it matters for aging. Brain Imaging Behav 8:274283.

Grady CL, Garrett DD (2018) Neuroimage Brain signal variability is modulated as a function of internal and external demand in younger and older adults. Neuroimage 169:510-523.

Guitart-masip M, Salami A, Garrett D, Rieckmann A, Lindenberger U, Bäckman L (2016) BOLD Variability is related to dopaminergic neurotransmission and cognitive aging. Cereb Cortex 26:2074-2083.

Haxby JV, Grady CL, Horwitz B, Ungerleider LG, Mishkin M, Carson RE, Herscovitch P, Schapiro MB, Rapoport SI (1991) Dissociation of object and spatial visual processing pathways in human extrastriate cortex. Proc Natl Acad Sci U S A 88:1621-1625.

Jack Jr CR, Holtzman DM (2013) Biomarker modeling of Alzheimer's disease. Neuron 80:1347-1358.

Jack Jr CR, Knopman DS, Jagust WJ, Petersen RC, Weiner MW, Aisen PS, Shaw LM, Vemuri P, Wiste HJ, Weigand SD, Lesnick TG, Pankratz VS, Donohue MC, Trojanowski JQ (2013) Tracking pathophysiological processes in Alzheimer's disease: an updated hypothetical model of dynamic biomarkers. The Lancet Neurology 12:207-216.

Jenkinson M, Bannister P, Brady M, Smith S (2002) Improved optimization for the robust and accurate linear registration and motion correction of brain images. Neuroimage 17:825-841.

Julayanont $\mathrm{P}$, Brousseau M, Chertkow $\mathrm{H}$, Phillips $\mathrm{N}$, Nasreddine ZS (2014) Montreal Cognitive Assessment Memory Index Score (MoCA-MIS) as a predictor of conversion from mild cognitive impairment to Alzheimer's disease. J Am Geriatr Soc 62:679-684.

Kielar A, Deschamps T, Chu RKO, Jokel R, Khatamian YB, Chen JJ, Meltzer JA (2016) Identifying dysfunctional cortex: dissociable effects of stroke and aging on resting state dynamics in MEG and fmri. Front Aging Neurosci 8:40.

Markwick A, Zamboni G, De Jager CA (2012) Profiles of cognitive subtest impairment in the Montreal Cognitive Assessment (MoCA) in a research cohort with normal Mini-Mental State Examination (MMSE) scores. J Clin Exp Neuropsychol 34:750-757.

Mclntosh AR, Lobaugh NJ (2004) Partial least squares analysis of neuroimaging data: applications and advances. Neuroimage 23: S250-S263.

Mclntosh AR, Mišić B (2013) Multivariate statistical analyses for neuroimaging data. Annu Rev Psychol 64:499-525.

Moscovitch M, Cabeza R, Winocur G, Nadel L (2016) Episodic memory and beyond: the hippocampus and neocortex in transformation. Annu Rev Psychol 67:105-134.

Nasreddine ZS, Phillips NA, Bedirian V, Charbonneau S, Whitehead V, Collin I, Cummings JL, Chertkow H (2005) The Montreal Cognitive Assessment, MoCA: a brief screening tool for mild cognitive impairment. J Am Geriatr Soc 53:695e699.

Newsome RN, Pun C, Smith VM, Ferber S, Barense MD (2013) Neural correlates of cognitive decline in older adults at-risk for developing $\mathrm{MCl}$ : evidence from the CDA and P300. Cogn Neurosci 4:152-162.

Nomi JS, Bolt TS, Ezie C, Uddin LQ, Heller AS (2017) Moment-tomoment BOLD signal variability reflects regional changes in neural flexibility across the lifespan. J Neurosci 37:5539-5548.

Olsen RK, Nichols EA, Chen J, Hunt JF, Glover GH, Gabrieli JDE, Wagner AD (2009) Performance-related sustained and anticipatory activity in human medial temporal lobe during delayed match-tosample. J Neurosci 29:11880-11890.

Olsen RK, Palombo DJ, Rabin JS, Levine B, Ryan JD, Rosenbaum RS (2013) Volumetric analysis of medial temporal lobe subregions in developmental amnesia using high-resolution magnetic resonance imaging. Hippocampus 23:855-860.

Olsen RK, Yeung LK, Noly-Gandon A, D'Angelo MC, Kacollja A, Smith VM, Ryan JD, Barense MD (2017) Human anterolateral entorhinal cortex volumes are associated with cognitive decline in aging prior to clinical diagnosis. Neurobiol Aging 57:195-205.

Osterreith PA (1944) Le test de copie d'une figure complex: contribution à l'étude de la perception et de la memoir. Arch Psychol 30:206-356.

Palombo DJ, Amaral RSC, Olsen RK, Muller DJ, Todd RM, Anderson AK, Levine B (2013) KIBRA polymorphism is associated with individual differences in hippocampal subregions: evidence from anatomical segmentation using high-resolution MRI. J Neurosci 33:13088-13093.

Perry A, Wen W, Kochan NA, Thalamuthu A, Sachdev PS, Breakspear M (2017) The independent influences of age and education on functional brain networks and cognition in healthy older adults. Hum Brain Mapp 38:5094-5114.

Petracca M, Saiote C, Bender HA, Arias F, Farrell C, Magioncalda P, Martino M, Miller A, Northoff G, Lublin F, Inglese M (2017) 
Synchronization and variability imbalance underlie cognitive impairment in primary-progressive multiple sclerosis. Sci Rep 7:46411-46412.

Phan KL, Wager T, Taylor SF, Liberzon I (2002) Functional neuroanatomy of emotion: a meta-analysis of emotion activation studies in PET and fMRI. Neuroimage 16:331-348.

Protzner AB, Kovacevic N, Cohn M, Mcandrews MP (2013) Characterizing functional integrity: intraindividual brain signal variability predicts memory performance in patients with medial temporal lobe epilepsy. J Neurosci 33:9855-9865.

Reitan RM, Wolfson D (1985) The Halstead-Reitan neuropsychological test battery: theory and clinical interpretation, Vol. 4. Tucson, AZ; Neuropsychology.

Ryan JD, Kacollja A, D'Angelo MC, Newsome RN, Gardner S, Rosenbaum RS (2019) Existing semantic knowledge provides a schematic scaffold for inference in early cognitive decline, but not in amnestic MCl. Cognitive Neuropsychology. Cogn Neuropsychol. doi: 10.1080/02643294.2019.

Scarapicchia V, Mazerolle EL, Fisk JD, Ritchie LJ, Gawryluk JR (2018) Resting state BOLD variability in Alzheimer's disease: a marker of cognitive decline or cerebrovascular status? Front Aging Neurosci 10:39.

Smith SM (2002) Fast robust automated brain extraction. Hum Brain Mapp 17:143-155.

Smith SM, Jenkinson M, Johansen-Berg H, Rueckert D, Nichols TE, Mackay CE, Watkins KE, Ciccarelli O, Cader MZ, Matthews PM, Behrens TEJ (2006) Tract-based spatial statistics: voxelwise analysis of multi-subject diffusion data. Neuroimage 31:1487-1505.

Steriade M, Llinás RR (1988) The functional states of the thalamus and the associated neuronal interplay. Physiol Rev 68:649-742.

Tabachnick BG, Fidell LS, (2007) Using multivariate statistics 5th ed. Allyn \& Bacon, Boston, MA.

Tsvetanov KA, Henson RNA, Tyler LK, Davis SW, Shafto MA, Taylor JR, Rowe JB (2015) The effect of ageing on fMRI: correction for the confounding effects of vascular reactivity evaluated by joint fMRI and MEG in 335 adults. Hum Brain Mapp 36:2248-2269.

Tsvetanov KA, Henson RN, Jones PS, Mutsaerts HJ, Fuhrmann D, Tyler LK, Rowe JB; Cam-CAN (2019) The effects of age on resting- state BOLD signal variability is explained by cardiovascular and neurovascular factors. BioRxiv. https://doi.org/10.1101/836619.

Turner BO, Lopez B, Santander T, Miller MB (2015) One dataset, many conclusions: BOLD variability's complicated relationships with age and motion artifacts. Brain Imaging Behav 9:115-127.

Tzourio-Mazoyer N, Landeau B, Papathanassiou D, Crivello F, Etard O, Delcroix N, Mazoyer B, Joliot M (2002) Automated anatomical labeling of activations in SPM using a macroscopic anatomical parcellation of the MNI MRI single-subject brain. Neuroimage 15:273-289.

van den Heuvel MP, Sporns O (2011) Rich-club organization of the human connectome. J Neurosci 31:15775-15786.

Warrington EK, James M (1991) A new test of object decision: 2D silhouettes featuring a minimal view. Cortex 27:377-383.

Wechsler D (1999) Manual for the Wechsler abbreviated intelligence scale (WASI). San Antonio: Psychological.

Wechsler D (2009) Wechsler memory scale (WMS-iv). New York: Psychological.

Wechsler D, Coalson D, Raiford S (2008) WAIS-iv: Wechsler adult intelligence scale. San Antonio: Pearson.

Yeung LK, Olsen RK, Bild-Enkin HEP, D’Angelo MC, Kacollja A, McQuiggan DA, Keshabyan A, Ryan JD, Barense MD (2017) Anterolateral entorhinal cortex volume predicted by altered intraitem configural processing. J Neurosci 37:5527-5538.

Yushkevich PA, Amaral RS, Augustinack JC, Bender AR, Bernstein JD, Boccardi M, Bocchetta M, Burggren AC, Carr VA, Chakravarty MM, Chételat G, Daugherty AM, Davachi L, Ding SL, Ekstrom A, Geerlings MI, Hassan A, Huang Y, Iglesias JE, La Joie R, et al. (2015) Quantitative comparison of 21 protocols for labeling hippocampal subfields and parahippocampal subregions in in vivo MRI: towards a harmonized segmentation protocol. Neuroimage 111:526-541.

Zöller D, Schaer M, Scariati E, Padula MC, Eliez S, Van De Ville D (2017) Disentangling resting-state BOLD variability and PCC functional connectivity in 22q11.2 deletion syndrome. Neuroimage 149:85-97. 